Mon. Not. R. Astron. Soc. 000,16(2014) Printed 8 May $2018 \quad$ (MN LATEX style file v2.2)

\title{
A Strange Dwarf Scenario for the Formation of the Peculiar Double White Dwarf Binary SDSS $\mathrm{J} 125733.63+542850.5$
}

\author{
Long Jiang ${ }^{1,2}$, Wen-Cong Chen ${ }^{1,2 \star}$, Xiang-Dong $\mathrm{Li}^{2,3} \dagger$ \\ 1 School of Physics, Shangqiu Normal University, Shangqiu 476000, China; \\ 2 Key Laboratory of Modern Astronomy and Astrophysics (Nanjing University), Ministry of Education, Nanjing 210093, China; \\ 3 Department of Astronomy, Nanjing University, Nanjing 210046, China
}

\begin{abstract}
The Hubble Space Telescope observation of the double white dwarf (WD) binary SDSS J125733.63+542850.5 reveal that the massive WD has a surface gravity $\log g_{1} \sim 8.7$ (which implies a mass of $M_{1} \sim 1.06 \mathrm{M}_{\odot}$ ) and an effective temperature $T_{1} \sim 13000 \mathrm{~K}$, while the effective temperature of the low-mass WD $\left(M_{2}<0.24 \mathrm{M}_{\odot}\right)$ is $T_{2} \sim 6400 \mathrm{~K}$. Therefore, the massive and the low-mass WDs have a cooling age $\tau_{1} \sim 1 \mathrm{Gyr}$ and $\tau_{2} \geqslant 5$ Gyr, respectively. This is in contradiction with traditional binary evolution theory. In this Letter, we propose a strange dwarf scenario to explain the formation of this double WD binary. We assume that the massive WD is a strange dwarf originating from a phase transition in a $\sim 1.1 \mathrm{M}_{\odot} \mathrm{WD}$, which has experienced accretion and spindown processes. Its high effective temperature could arise from the heating process during the phase transition. Our simulations suggest that the progenitor of SDSS $\mathrm{J} 125733.63+542850.5$ can be a binary system consisting of a $0.65 \mathrm{M}_{\odot} \mathrm{WD}$ and a $1.5 \mathrm{M}_{\odot}$ main sequence star in a 1.492 day orbit. Especially, the secondary star (i.e., the progenitor of the low mass WD) is likely to have an ultra-low metallicity of $Z=0.0001$.
\end{abstract}

Key words: stars: evolution - evolution: phase transition - double white dwarf: individual SDSS J125733.63+542850.5

\section{INTRODUCTION}

As the end products of binary evolution, double white dwarf (WD) binaries are good probes for testing stellar and binary evolutionary theory (Marsh et al. 1995; Toonen et al. 2014). Especially, they are thought to be progenitors of Type Ia supernovae (Iben \& Tutukov 1984; Webbink 1984), AM CVn systems (Camilo et al. 1996; Kilic et al. 2014), and R CrB stars (Webbink 1984). Furthermore, close double WDs are believed to be the main Galactic gravitational sources in the frequency range of $10^{-4}$ to $0.1 \mathrm{~Hz}$, which will be detected by the Laser Interferometer Space Antenna detector (Hils et al. 1990; Nelemans et al. 2001; Hermes et al. 2012).

Based on the Sloan Digital Sky Survey (SDSS; Eisenstein et al. (2006); York et al. (2000)) subspectra, the double WD binary SDSS J125733.63+542850.5 (hereafter J1257) was first discovered by the Sloan WD Radial velocity Mining Survey (Badenes et al. 2009). Its radial velocity variations with a semi-amplitude of $323 \mathrm{~km} \mathrm{~s}^{-1}$ were interpreted to originate from a $0.9 \mathrm{M}_{\odot} \mathrm{WD}$ while the companion was

\footnotetext{
* E-mail:chenwc@pku.edu.cn

† E-mail:lixd@nju.edu.cn
}

suggested to be a neutron star or black hole (Badenes et al. 2009 ). The two distinct components were revealed in the spectra of $B$ and $R$ band spectroscopy, and the Balmer lines with deep radial velocity variable were identified to come from a cool, extremely low-mass WD with mass less than $0.3 \mathrm{M}_{\odot}($ Kulkarni \& van Kerkwiik 2010; Marsh et al. 2011).

Recently, Bours et al. (2015) fit both the Hubble Space Telescope Cosmic Origins Spectrograph and the Space Telescope Imaging Spectrograph spectra, and the SDSS ugriz flux with a Markov Chain Monte Carlo approach. Their results indicate that the massive component has a surface gravity $\log g_{1} \sim 8.73 \pm 0.05$, and an effective temperature $T_{1} \sim 13030 \pm 70 \mathrm{~K}$. Detailed evolutionary models reveal the mass to be $M_{1}=1.06 \pm 0.05 \mathrm{M}_{\odot}$, and a corresponding cooling age of $\tau_{1}=1.0 \mathrm{Gyr}$ or $1.2 \mathrm{Gyr}$ for carbon/oxygen and oxygen/neon WD models respectively (Kowalski \& Saumon 2006; Althaus et al. 2007; Tremblay et al. 2011). However, the low-mass WD with $M_{2}<0.24 \mathrm{M}_{\odot}$ has a temperature of $T_{2} \sim 6400 \mathrm{~K}$ and a cooling age of $\tau_{2} \geqslant 5$ Gyr (Marsh et al. 2011; Bours et al. 2015). The two cooling ages are in contradiction with binary stellar evolutionary theory. The progenitor of the low-mass He WD probably had a mass of $1-2 \mathrm{M}_{\odot}$ (Istrate et al. 2014), and should have evolved much 
more slowly than $5-6 \mathrm{M} \odot$ progenitor of the more massive WD. After the formation of the low-mass WD, CNO flashes could cause it to fill its Roche lobe, and accretion heating would alter the thermal structure of the massive WD with a duration of $\sim 10^{6}$ yr (Bildsten et al. 2006). However, this mass transfer timescale $(\sim 100 \mathrm{yr})$ is too short to influence the cooling history of the massive WD.

In this paper, we propose that the difference in the cooling ages originate from the heating process during the formation of a strange dwarf. We describe the strange dwarf scenario in section 2 . In section 3 , we discuss the changes of the orbital parameters during phase transition and the possible spin-down process of the massive WD. Employing the MESA code, we simulate the evolutionary history of J1257 in section 4. A brief summary and discussion are presented in section 5 .

\section{STRANGE DWARF SCENARIO}

Based on the hypothesis that the strange quark matter may be the most stable state of matter, Witten (1984) proposed that the pulsars may be strange quark stars (SSs) rather than neutron stars (NSs). Following this idea, the concept of strange dwarfs (SDs) was introduced by Glendenning et al. $(1995 \mathrm{a}$ b) as strange counterpart of WDs. They pointed out that the inner density of ordinary stable WDs is always below critical density $\rho_{\mathrm{c}} \sim 10^{9} \mathrm{~g} \mathrm{~cm}^{3}$, which is the central density of a maximum-mass $\left(\sim 1 \mathrm{M}_{\odot}\right)$ WD (Baym et al. 1971). The structure and thermal evolution of SDs with different masses have been studied by Benvenuto \& Althaus (1996) in detail. Their computation indicates that the thermal evolution of SD with mass larger than $1 \mathrm{M}_{\odot}$ is similar to that of a WD with the same mass.

Following these studies, we assume that if the central density of a WD exceeds a critical density, a strange quark core will emerge in the center region and the star evolves to be a SD. During this phase transition (PT, hereafter) process the mass of star will decrease slightly (about several percent). Part of them will translate to the binding energy of the more compact SD, and the other is lost from the star. The energy transportation during the process may heat the star and result in a hotter SD.

Under the assumptions mentioned above, we outline the evolutionary stages of J1257 as follows, which is also illustrated in Fig. 1.

- We start from a compact binary (with an orbital period $P_{i} \sim 1.5$ days), which consists of a white dwarf with $M_{1} \sim$ $0.6-0.8 \mathrm{M}_{\odot}$ and a main sequence star with $M_{2} \sim 1.5 \mathrm{M}_{\odot}$.

- Roche lobe overflow. The WD accretes around $0.3-$ $0.5 \mathrm{M}_{\odot}$ material from the donor star, and spins up to nearly breakup rotation. The spin-up process would also significantly broaden the hydrogen line profiles, as reported by Kulkarni \& van Kerkwiik (2010).

- Formation of the second WD. The mass transfer terminates and the secondary star evolves into a low-mass WD. The central density of the primary WD, which is centrifugally diluted by the fast spinning due to accretion starts to spin down. Mean while the second WD gradually cools.

- PT. After several Gyr, the massive WD's spin down causes its density to be above the critical density, thus PT

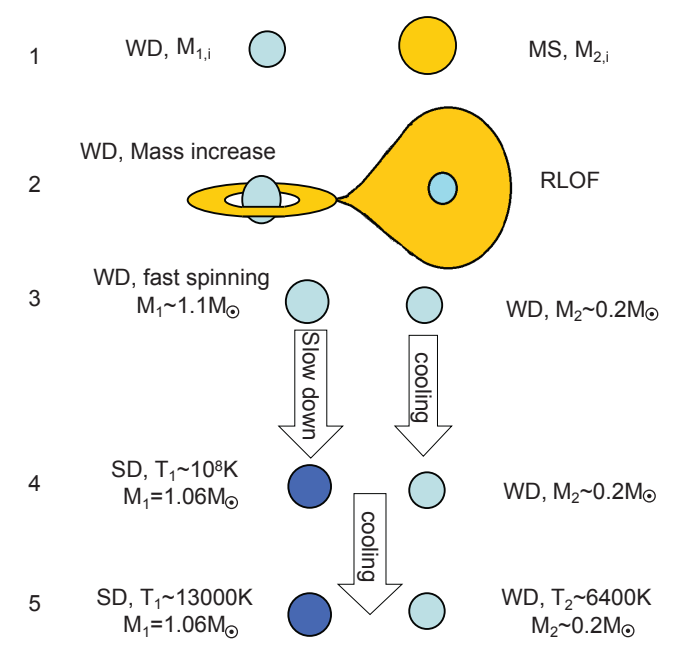

Figure 1. Illustration of our strange dwarf evolution from the compact white dwarf binary to the present observed system $\mathrm{J} 1257$.

takes place in its core and a nascent $\mathrm{SD}$ is heated up to $10^{8} \mathrm{~K}$.

- Cooling of the massive SD. According to Benvenuto \& Althaus (1996), the cooling process of the SD is similar to a normal WD with the same mass.

The temperature of the nascent SD can be estimated as follows. Assuming that the difference between the gravitational masses of a WD and a SD with the same quark number is $\Delta M$, and that a fraction $\alpha(<1)$ of the rest energy of the mass loss during PT was used to heat the nascent $\mathrm{SD}$, the thermal energy received by the $\mathrm{SD}$ is:

$$
Q=\alpha \Delta M c^{2} \text {. }
$$

The internal energy of the SD with a temperature $T$ can be estimated as:

$$
U_{\mathrm{SD}}=\frac{3}{2} k_{\mathrm{B}} N T
$$

where $k_{\mathrm{B}}$ is Boltzmann's constant and $N=M_{\mathrm{SD}} / A m_{\mathrm{u}}$ is the total number of quarks and electrons. Here $M_{\mathrm{SD}}$ is the mass of the SD, $m_{\mathrm{u}}$ is the atomic mass unit, $A$ is the relative particle mass. Following Alcock et al. (1986), we assume that the SD is composed by roughly equal numbers of up, down and strange quarks and a small number (ignorable) electrons. Considering that the mass of SD decreased a little during PT while the quark number keeps constant, we take $A \sim 0.3$.

Since the effective temperature of the WD before PT was much lower than the nascent $\mathrm{SD}$, the internal energy of WD $U_{\mathrm{WD}}\left(\ll U_{\mathrm{SD}}\right)$ is ignorable. Because $Q=U_{\mathrm{SD}}-U_{\mathrm{WD}} \approx$ $U_{\mathrm{SD}}$, the temperature of the nascent $\mathrm{SD}$ can be written as:

$$
T=\frac{2}{3} \frac{\alpha \Delta M c^{2}}{k_{\mathrm{B}} N}=\frac{2}{3} \frac{\alpha \Delta M c^{2} A m_{\mathrm{u}}}{k_{\mathrm{B}} M_{\mathrm{SD}}} .
$$

PT between NS and SS has been extensively investigated for different equations of state. Several works suggested that the difference between the gravitational masses a NS and a SS with the same baryon number is roughly $M_{\mathrm{NS}}-M_{\mathrm{SS}} \approx 0.15 \mathrm{M}_{\odot}$ for a NS with mass $\sim 1.5 \mathrm{M}_{\odot}$ (e.g., Bombaci \& Datta 2000; Drago et al. 2007; 
Marquez \& Menezes 2017)1 1 . Considering the difference in the mass and compactness between a WD and a NS, in this work we take $\Delta M \sim 0.05 \mathrm{M}_{\odot}$. Considering that most of the energy liberated during PT is assumed to be taken away by neutrinos (and anti-neutrinos), similar as in supernova explosions (Kippenhahn et al. 2012), and only a small fraction is used to heat the nascent SD, we set $\alpha \sim 0.001$ as lower limit. Taking $A \sim 0.3$, and $M_{\mathrm{SD}}=1.05 \mathrm{M}_{\odot}$, the nascent SD had an initial effective temperature $T \simeq 10^{8} \mathrm{~K}$. In comparison, the temperature of a $1.0 \mathrm{M}_{\odot} \mathrm{WD}$ is $<10^{7} \mathrm{~K}$.

\section{ORBITAL CHANGE DURING PT AND THE SPIN DOWN OF PROGENITOR WD}

\subsection{Orbital change during PT}

We first discuss the influence of PT on the eccentricity of the binary. Considering that the PT in the core of WD took place quickly, and a kick velocity $V_{\mathrm{k}}$ was imparted to the new born $\mathrm{SD}$, one can solve the orbital parameters during PT following Shao \& Li (2016). Due to the mass transfer with a long duration, the orbit of the binary before PT can be thought to be circular. Setting $\phi$ to be the positional angle of $V_{\mathrm{k}}$ with respect to the pre-PT orbital plane and $\theta$ the angle between $V_{\mathrm{k}}$ and the pre-PT orbital velocity $V_{0}$ $\left(=\left(2 \pi G M_{0} / P_{\text {orb }, 0}\right)^{1 / 3}\right)$, the ratio between the semi-major axes before and after PT is (Hills 1983; Dewi \& Pols 2003):

$$
\frac{a_{0}}{a}=2-\frac{M_{0}}{M_{0}-\Delta M}(1+\nu+2 \nu \cos \theta),
$$

where $\nu=V_{\mathrm{k}} / V_{0}$, and $M_{0}$ and $P_{\text {orb, } 0}$ are the total mass of the binary and orbital period of the binary before PT, respectively. Under the influence of mass loss and kick, the eccentricity after PT can be written as Hills 1983; Dewi \& Pols 2003):

$$
\begin{aligned}
1-e^{2}=\frac{a_{0} M_{0}}{a\left(M_{0}-\Delta M\right)}[ & +2 \nu \cos \theta \\
& \left.+\nu^{2}\left(\cos ^{2} \theta+\sin ^{2} \theta \sin ^{2} \phi\right)\right] .
\end{aligned}
$$

Taking $M_{0}=M_{2}+M_{1}=1.3 \mathrm{M}_{\odot}, \Delta M=0.05 \mathrm{M}_{\odot}$, $P_{\text {orb }, 0}=0.22$ day, we simulated the possibility of small eccentricities $(e<0.01)$ after $\mathrm{PT}$ for different $V_{\mathrm{k}}$ in the range of $0-50 \mathrm{~km} \mathrm{~s}^{-1}$. For each $V_{\mathrm{k}}$, we set $10^{7}$ independent random values for $\cos \theta$ and $\phi$ of uniform distribution in the interval of -1 to 1 and 0 to $\pi$, respectively. According to the observations of Badenes et al. (2009) and Marsh et al. (2011), the current orbit of J1257 is circular and a WD binary with $e<0.01$ could evolve into a circular orbit on a timescale of $\sim 1$ Gyr. Fig. 2 shows the possibility distribution for the eccentricity less than 0.01 with different kick velocities. When $V_{\mathrm{k}} \leqslant 5 \mathrm{~km} \mathrm{~s}^{-1}$ and $V_{\mathrm{k}} \geqslant 50 \mathrm{~km} \mathrm{~s}^{-1}$, the possibilities with $e<0.01$ are less than $0.1 \%$ and $0.2 \%$, respectively. However, the relevant possibility is $\geqslant 1 \%$ when $6 \leqslant V_{\mathrm{k}} \leqslant 20 \mathrm{~km} \mathrm{~s}^{-1}$. Especially, the relevant possibility is as high as $10 \%$ for a kick velocity range of $8-9 \mathrm{~km} \mathrm{~s}^{-1}$. Similar to accretion induced collapse of neutron star (Hurley et al. 2010), the nascent SD should obtain a low kick velocity.

1 Lower value is also possible, for example, the gravitational mass between NS and hyperon star given by Schaffner-Bielich et al. (2002) is $\sim 0.03 \mathrm{M}_{\odot}$.

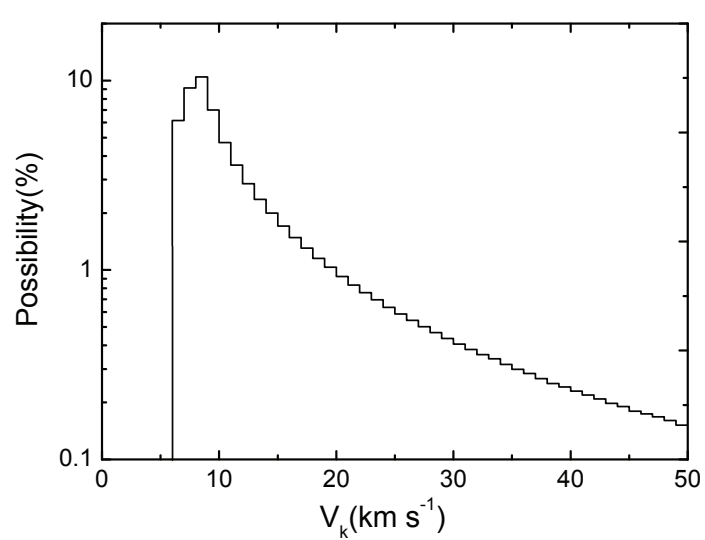

Figure 2. Possibility distribution for the eccentricity less than 0.01 under different values of the kick velocity.

Therefore, PT process has a relatively large possibility to result in a nearly circular orbit.

According to the relation between the pre-PT and the post-PT orbital separation $a_{0} /(1+e) \leqslant a \leqslant a_{0} /(1-$ e) (Flannerv \& van den Heuvel 1975$)$, one can derive the change of orbital period is $\leqslant 2 \%$ when $e<0.01$. Since the changes are relatively small, we ignore the orbital change of the binary during PT in our simulation.

\subsection{Spin down of massive WD}

Similar to pulsars, we consider the spin-down of WD with an angular velocity $\Omega=2 \pi / P_{\mathrm{s}}$ is dominated by magnetic dipole radiation 2 , and the energy loss rate is:

$$
\dot{E}_{\mathrm{d}}=-\frac{2}{3 c^{3}} \mu^{2} \Omega^{4}
$$

where $\mu=B R^{3}=B_{7} R_{9}^{3} \times 10^{34} \mathrm{G} \mathrm{cm}^{3}$ is the magnetic dipole moment, and $B_{7}$ is the surface magnetic field in units of $10^{7} \mathrm{G}$, and $R_{9}$ is the radius in units of $10^{9} \mathrm{~cm}$ of the WD, respectively.

The rotational energy of WD changes at a rate:

$$
\dot{E}_{\mathrm{s}}=I \Omega \dot{\Omega}
$$

where $I \sim M R^{2} \approx 10^{51} R_{9}^{2} \mathrm{~g} \mathrm{~cm}^{2}$ is the moment of inertia of WD. If we assume that the braking torque of the WD fully originate from the magnetic dipole radiation, the spin period of the WD changes at a rate:

$$
\dot{P}_{\mathrm{s}}=\frac{8 \pi^{2}}{3 c^{3}} \frac{\mu^{2}}{I P_{\mathrm{s}}}=K / P_{\mathrm{s}}
$$

where $K=8 \pi^{2} \mu^{2} / 3 c^{3} I \sim B_{7}^{2} R_{9}^{4} \times 10^{-13} \mathrm{~s}$.

With simple integration, one can get the spin-down timescale of the WD from the initial spin period $P_{\mathrm{s}, 0}$ to the spin period of PT $P_{\mathrm{s}}$ :

$$
\tau_{\mathrm{SD}}=\frac{P_{\mathrm{s}}^{2}-P_{\mathrm{s}, 0}^{2}}{2 K} \approx \frac{P_{\mathrm{s}}^{2}-P_{\mathrm{s}, 0}^{2}}{6 B_{7}^{2} R_{9}^{4}} \mathrm{Myr} .
$$

\footnotetext{
2 Gravitational wave radiation might be an efficient mechanism extracting angular momentum from fast rotating WDs due to $\mathrm{r}$ mode instability in a short timescale $\left(<10^{8} \mathrm{yr}\right.$, Yoon \& Langer 2004)
} 


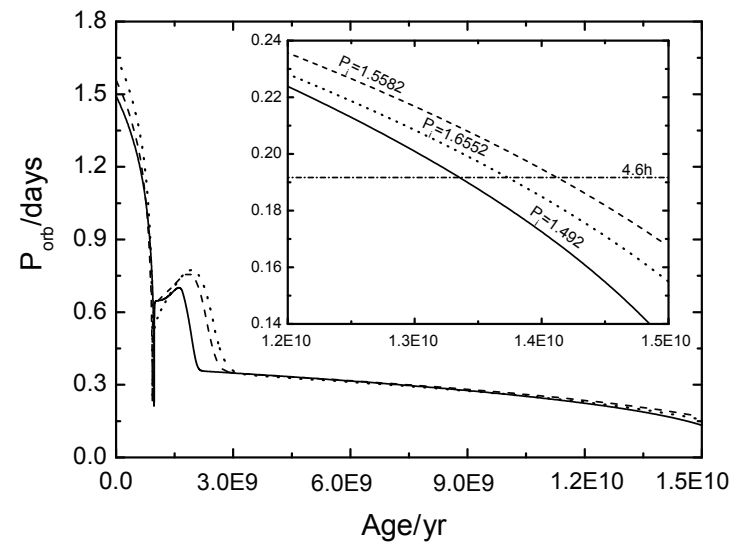

Figure 3. Evolution of orbital period for WD binaries including a $1.5 \mathrm{M}_{\odot}$ donor star and a $0.65 \mathrm{M}_{\odot} \mathrm{WD}$. The solid, dashed and dotted curves represent the metallicity $z=0.0001,0.0005$ and 0.001 respectively. Numbers inside the curves denote the initial orbital periods.

Based on the theory of accretion disk-magnetic field interaction developed by Ghosh \& Lamb (1979), Kulkarni \& van Kerkwiik (2010) inferred that the magnetic field of J1527 was $\sim 10^{5} \mathrm{G}$ when it spin up to its current spin period of $\sim 60$ s. However, Cumming (2002) showed that rapid accretion could reduce the field strength at the surface of the accreting WD because the field is advected into the interior by the accretion flow. Therefore, many nonmagnetic WDs ( $B \lesssim 10^{5} \mathrm{G}$ ) may have submerged magnetic fields when they were accreting at rates greater than the critical rate $\dot{M}_{\mathrm{cr}}=1-5 \times 10^{-10} \mathrm{M}_{\odot} \mathrm{yr}^{-1}$, and the magnetic field would re-emerge when the mass transfer terminates, and the re-emergence timescale of the field is:

$$
\tau_{\mathrm{re}} \simeq 300 \times\left(\frac{\Delta M_{\mathrm{acc}}}{0.1 \mathrm{M}_{\odot}}\right)^{7 / 5} \mathrm{Myr}
$$

where $\Delta M_{\text {acc }}$ is the accreted mass of the WD. Based on the model of Cumming (2002) for magnetic field evolution, we propose that the surface field of the more massive WD in $\mathrm{J} 1257$ decreased from $\sim 10^{7} \mathrm{G}$ to $\sim 10^{5} \mathrm{G}$ due to rapid accretion. According to the simulation in the next section, the accreted mass of the massive WD is $\Delta M_{\text {acc }} \approx 0.45 \mathrm{M}_{\odot}$, so the re-emergence timescale $\tau_{\text {re }} \approx 2.5 \mathrm{Gyr}$.

Taking $P_{\mathrm{s}, 0}=10 \mathrm{~s}$ (close to breakup rotation), $P_{\mathrm{s}}=$ $60 \mathrm{~s}, B_{7}=1$, and $R_{9}=0.6$, one can derive the spindown timescale of the massive WD is about 4.5 Gyr. Considering the re-emergence timescale of the magnetic field $\tau_{\text {re }} \approx 2.5$ Gyr, the total duration of 7 Gyr before the PT for the massive WD is approximately in agreement with the cooling age of the low mass WD.

\section{NUMERICAL SIMULATION}

Using the MESA module (Paxton et al. 2015), we have simulated the evolution of the compact WD binary consisting of a WD and a main sequence star, to test whether it is possible to reproduce the characteristics of J1257. According to the estimation in the previous section, the orbital change

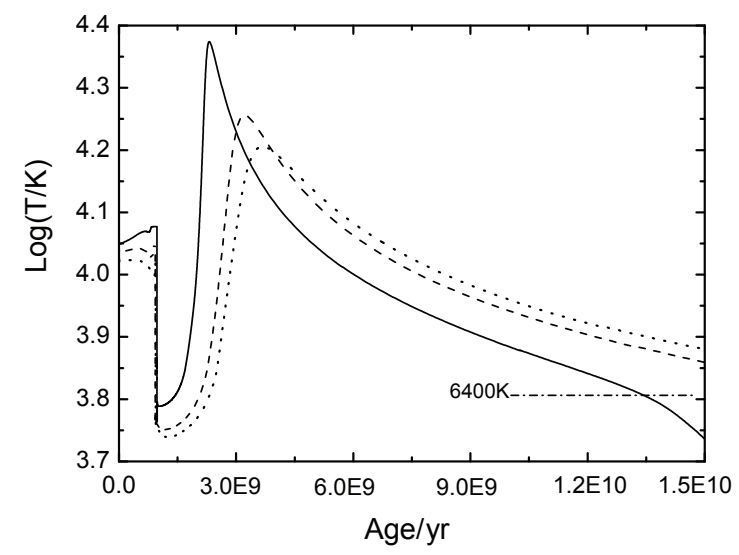

Figure 4. Evolutionary tracks of the effective temperature of donor star for WD binaries including a $1.5 \mathrm{M}_{\odot}$ donor star and a $0.65 \mathrm{M}_{\odot}$ WD. Three cases are same to Figure 3 .

during PT can be neglected while the mass growth of the WD is considered.

For an accreting WD, hydrogen and helium shell flashes always trigger nova outbursts, which blow off the accreted matter and even result in convective dredge-up. Therefore, the mass accumulation efficiency for accreting hydrogen should be less than 1 . During the mass transfer, the mass growth rate of the accreting WD is described as follows:

$$
\dot{M}_{1}=\eta_{\mathrm{He}} \eta_{\mathrm{H}}\left|\dot{M}_{2}\right|
$$

where $\dot{M}_{2}$ is the mass transfer rate of the donor star, $\eta_{\mathrm{H}}$ and $\eta_{\mathrm{He}}$ is the accumulation efficiencies during hydrogen burning and helium burning, respectively.

For the accumulation efficiency of hydrogen, a prescription given by Hachisu et al. (1999) and Han \& Podsiadlowski (2004) was adopted, i.e.

$$
\eta_{\mathrm{H}}= \begin{cases}\dot{M}_{\mathrm{cr}} /\left|\dot{M}_{2}\right| & \left|\dot{M}_{2}\right|>\dot{M}_{\mathrm{cr}}, \\ 1 & \dot{M}_{\mathrm{cr}}>\left|\dot{M}_{2}\right|>0.125 \dot{M}_{\mathrm{cr}} \\ 0 & \left|\dot{M}_{2}\right|<0.125 \dot{M}_{\mathrm{cr}} .\end{cases}
$$

In equation (12), $\dot{M}_{\text {cr }}$ is a critical mass-accretion rate:

$$
\dot{M}_{\mathrm{cr}}=5.3 \times 10^{-7} \frac{1.7-X}{X}\left(M_{1}-0.4\right) \mathrm{M}_{\odot} \mathrm{yr}^{-1},
$$

where $X$ is the mass abundance of hydrogen in the accreted matter.

For the accumulation efficiency $\eta_{\mathrm{He}}$ during helium burning, the prescriptions given by Kato \& Hachisu (2004) was adopted. The mass loss $\left(1-\eta_{\mathrm{H}} \eta_{\mathrm{He}}\right) \dot{M}_{2}$ during hydrogen and helium burning is assumed to be ejected in the vicinity of the WD in the form of isotropic winds, carrying away the specific angular momentum of the WD (Hachisu et al. 1996; Soberman et al. 1997). In addition, we also consider angular momentum loss caused by gravitational radiation and magnetic braking (with $\gamma=3.0$, Rappaport et al. (1983); Paxton et al. (2015)).

To study the progenitor properties of J1257, we simulated the evolution of a large number of WD binaries. The relevant binaries would be thought to be the progenitor candidates of J1257 if the following three conditions are satisfied: (1) the current orbital period is $4.6 \mathrm{hr}$ when the age 

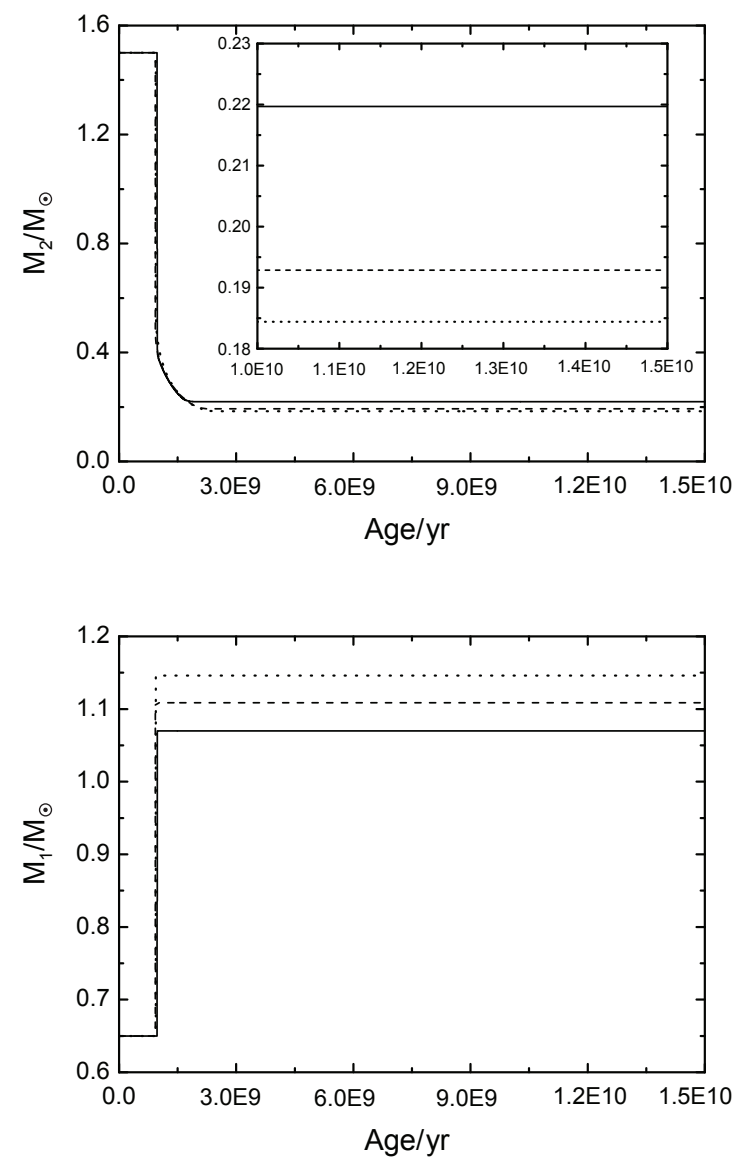

Figure 5. Evolution of the donor-star mass (top panel) and the WD mass (bottom panel) WD binaries including a $1.5 \mathrm{M}_{\odot}$ donor star and a $0.65 \mathrm{M}_{\odot}$ WD. Three cases are same to Figure 3 .

of the system is within Hubble time; (2) the binary evolves into a detached system when the accreting WD's mass is about $1.05-1.15 \mathrm{M}_{\odot}$ (Because the WD must experience a long-term spin-down process, hence the donor star should not overflow its Roche lobe when the WD mass increases to $\left.1.05-1.15 \mathrm{M}_{\odot}\right)$; (3) the effective temperature of the donor star is near $6400 \mathrm{~K}$. Figures 2-4 show an example evolution in which the initial donor mass and the initial WD mass are $1.5 \mathrm{M}_{\odot}$, and $0.65 \mathrm{M}_{\odot}$, respectively. We change the initial metallicity in order to fit the observed parameters of J1257.

As shown in Fig. 3, because the material is transferred from the more massive donor star to the less massive WD, the orbital period firstly decreases. With the reversal of the mass ratio, the orbital period increases until the binary evolve into a detached system. Subsequently, the donor star gradually evolve into a WD and enters the cooling stage, and magnetic braking and gravitational radiation induce a compact double WDs binary. Once the mass transfer ceases, the massive WD spin down due to magnetic dipole radiation, then trigger PT during the cooling of the low-mass WD. The heating process during PT results in the formation of a hot SD. Similar to Fig. 4, the simulated effective temperatures of the low-mass WD are always higher than the observation in the Hubble time except for $z=0.0001$, in which the donor star orbits a WD with an initial orbital period of 1.492 days.
Fig. 5 shows the evolutionary tracks of the donor-star mass and the accreting WD's mass. It is clear that our simulated donor-star masses are consistent with the observed data for three different metallicities. In calculation, the donor star with higher metallicity would produce systems with lower mass secondary WDs. These difference should arise from the metallicity dependence of the stellar wind mass loss, which tend to reduce the stellar mass of donor star.

\section{SUMMARY AND DISCUSSION}

Assuming both WD and SD are different stages of stellar evolution, in this work we propose a SD scenario to interpret the puzzle of the cooling age of two WD in J1257. The massive WD is thought to be a SD originating from the $\mathrm{PT}$ of the 1.05-1.15 $\mathrm{M}_{\odot} \mathrm{WD}$, thus its higher effective temperature can be interpreted as a result of heating during PT. A simple estimation indicates that a mass loss $\Delta M \sim 0.05 \mathrm{M}_{\odot}$ during PT can heated the nascent SD up to $10^{8} \mathrm{~K}$. Based on these assumptions, we use the MESA code to simulate the evolution of a large number of WD binaries consisting of a $0.65 \mathrm{M}_{\odot} \mathrm{WD}$ and a $1.5 \mathrm{M}_{\odot}$ main-sequence star for different initial orbital period and metallicities. Our simulation indicate that metallicities have important influence on the effective temperature of the donor star. When $z=0.0001$, the calculate orbital period, the donor-star mass, and the effective temperature of the donor star are consistent with the observed data. Therefore, we propose that the PT of a massive WD may be responsible for the puzzling cooling age of two WDs. We expect further detailed multi-waveband observations for this source to obtain more precise constraints.

\section{ACKNOWLEDGMENTS}

This work was supported by the National Natural Science Foundation of China under grant number 11573016, 11733009, 11773015 and 11605110, the National Key Research and Development Program of China (2016YFA0400803), and the Program for Innovative Research Team (in Science and Technology) at the University of Henan Province.

\section{REFERENCES}

Alcock C., Farhi E., Olinto, A., 1986, ApJ, 310, 261

Althaus L. G., García-Berro E., Isern, J., Córsico A. H., Rohrmann R. D., 2007, A\&A, 465, 249

Badenes C., Mullally F., Thompson S. E., Lupton R. H., 2009, ApJ, 707, 971

Benvenuto O. G., Althaus L. G., 1996, ApJ, 462, 364

Baym G., Pethick C., Sutherland P., 1971, ApJ, 170, 299

Bildsten L., Townsley D. M., Deloye C. J., Nelemans G., 2006, ApJ, 640, 466

Breedt E., Gänsicke B. T., Marsh T. R., Steeghs D., Drake

A. J. Copperwheat C. M., 2012, MNRAS, 425, 2548

Bombaci I., Datta B., 2000, ApJL, 530, L69

Bours M.C.P., Marsh T.R., et al., 2015, MNRAS, 450, 3966

Cumming A., 2002, MNRAS, 333, 589

Dewi J.D.M., Pols, O. R., 2003, MNRAS, 344629

Drago A., Lavagno A., Parenti I., 2007, ApJ, 659, 1519 
Eisenstein D. J., et al., 2006, ApJS, 167, 40

Flannery B. P., van den Heuvel E. P. J., 1975, A\&A, 39, 61

Ghosh P., Lamb F. K., 1979, ApJ, 234, 296

Glendenning N. K., Kettner C., Weber F., 1995a, PhRvL, 74,3519

Glendenning N. K., Kettner C., Weber F., 1995b, ApJ, 450, 253

Hachisu I., Kato M., Nomoto K., 1996, ApJL, 470, L97

Hachisu I., Kato M., Nomoto K., Umeda H., 1999, ApJ, 519,314

Han Z., Podsiadlowski Ph., 2004, MNRAS, 350, 1301

Hermes J. J., et al., 2012, ApJ, 757, L21

Hills J.G., 1983, ApJ, 267, 322

Hils D., Bender P. L., Webbink R. F., 1990, ApJ, 360, 75

Hurley J. R., Tout C. A., Wickramasinghe D. T., et al., 2010, MNRAS, 402, 1437

Iben Jr., I., Tutukov A. V., 1984, ApJS, 54, 335

Istrate A. G., Tauris T. M., Langer N., 2014, A\&A, 571, A45

Kato M., Hachisu I., 2004, ApJ, 613, L129

Kilic M., et al., 2014b, MNRAS, 438, L26

Kippenhahn R., Weigert A., Weiss A., 2012, Stellar Structure and Evolution, (Springer)

Kowalski P. M., Saumon D., 2006, ApJ, 651, L137

Kulkarni S. R., van Kerkwijk M. H., 2010, ApJ, 719, 1123

Marquez K. D., Menezes D. P., 2017, arXiv:1709.07040

Marsh T. R., Dhillon V. S., Duck S. R., 1995, MNRAS, 275,828

Marsh T. R., Gänsicke B. T., Steeghs D., et al., 2011, ApJ, 736,95

Nelemans G., Yungelson L. R., Portegies Zwart S. F., 2001, A\&A, 375, 890

Paxton B., Marchant P., Schwab J., et al., 2015, ApJS, 220, 15

Rappaport S., Verbunt F., Joss P.C., 1983, ApJ, 275, 713

Schaffner-Bielich J., Hanauske M., Stöcker H., Greiner W., 2002, Phys. Rev. Lett., 89, 171101

Shao Y., Li X.-D., 2016, ApJ, 816, 45

Soberman G. E., Phinney E. S., van den Heuvel E. P. J., 1997, A\&A, 327, 620

Toonen S., Claeys J. S. W., Mennekens N., Ruiter A. J., 2014, A\&A, 562, A14

Tremblay P.-E., Bergeron P., Gianninas, A., 2011, ApJ, 730, 128

Verbunt F., Zwaan C., 1981, A\&A, 100, L7

Webbink R. F., 1984, ApJ, 277, 355

Witten, E., 1984, Phys. Rev. D, 30, 272

Yoon S. -C., Langer N., 2004, A\&A, 419, 623

York D. G., et al., 2000, AJ, 120, 1579

This paper has been typeset from a $\mathrm{TE}_{\mathrm{E}} \mathrm{X} / \mathrm{H} \mathrm{AT}_{\mathrm{E}} \mathrm{X}$ file prepared by the author. 